\title{
Epidemiología de la candidemia y sensibilidad in vitro frente a azoles, anfotericina $B$ y caspofungina en una institución de salud de Valledupar, Colombia
}

\author{
Epidemiology of candidemia and in vitro susceptibility to azoles, amphotericin B
} and caspofungin in a health institution of Valledupar, Colombia

Carmen A. Vides-Peña MSc', Nalleth D. Bolaño-Ardila MSc', Máryuris V. Vides-Peña MSc ${ }^{3}$, Susana B. Córdoba PhD ${ }^{4}$

Introducción: la candidemia es una enfermedad grave, con elevada morbi-mortalidad y cuyo tratamiento no siempre conduce a la cura. La distribución de especies de Candida y la sensibilidad antifúngica varía según el área geográfica, incluso entre centros de salud de una misma región. Objetivo: establecer la distribución de especies de Candida y su sensibilidad in vitro frente a diferentes antifúngicos. Materiales y métodos: se realizó un estudio descriptivo, prospectivo y transversal entre noviembre de 2013 y mayo de 2014 de casos de candidemias en una institución de salud de Valledupar, Colombia. Las Concentraciones Inhibitorias Mínimas (CIM) se determinaron según el protocolo estándar M27-A3-S4. Resultados: Se estudiaron 40 aislados clínicos de Candida spp. obtenidos de sangre (97,5\%) y médula ósea (2,5\%). Del total, 15 (37,5\%) fueron caracterizados como Candida tropicalis, 13 (32,5\%) del Complejo Candida albicans, cinco (1 2,5\%) del Complejo Candida glabrata, tres (7,5\%) como Candida guilliermondii, tres (7,5\%) del Complejo Candida parapsilosis y uno (2,5\%) como Candida krusei. No se observó resistencia a la anfotericina B ni al voriconazol en ninguno de los aislados, pero sí al fluconazol en uno (6,6\%) de Candida tropicalis y uno (33,3\%) del Complejo Candida parapsilosis y a la caspofungina en el aislado de Candida krusei y en uno (20\%) del Complejo Candida glabrata. Conclusiones: la epidemiología local de las levaduras causantes de candidemia mostró mayor prevalencia de especies no-albicans, entre las que se encontró resistencia a los antifúngicos evaluados, lo que es relevante para la elección e instauración de un tratamiento eficaz.

Palabras clave: Candida, candidemia, pruebas de sensibilidad microbiana, antifúngicos.

\footnotetext{
'Microbióloga, Esp. y MSc. en Microbiología Clínica. Grupo para el Estudio de la Resistencia Microbiana y la Microbiología Clínica en el Cesar-GREMIC. Facultad Ciencias de la Salud, Universidad Popular del Cesar. Valledupar, Colombia. Correo electrónico: carmenvides@unicesar.edu.co

${ }^{2}$ Microbióloga, Esp. y MSc. en Microbiología Clínica. Grupo para el Estudio de la Resistencia Microbiana y la Microbiología Clínica en el Cesar-GREMIC. Facultad Ciencias de la Salud, Universidad Popular del Cesar. Valledupar, Colombia.

3Microbióloga, MSc. en Microbiología Clínica. Grupo para el Estudio de la Resistencia Microbiana y la Microbiología Clínica en el Cesar-GREMIC. Facultad Ciencias de la Salud, Universidad Popular del Cesar. Valledupar, Colombia.

${ }^{4} \mathrm{MD}$ Veterinaria, Bacterióloga Clínica e Industrial, MSc. en Microbiología Molecular, PhD. en Bacteriología Clínica. Laboratorio de Antifúngicos, Departamento de Micología, Instituto Nacional de Enfermedades Infecciosas (INEI-ANLIS) "Dr. Carlos G. Malbrán". Ciudad Autónoma de Buenos Aires, Argentina.
}

Conflicto de intereses: las autoras declaran que no tienen conflicto de intereses Medicina \& Laboratorio 2015; 21: 255-266

Módulo 19 (Investigación), número 37. Editora Médica Colombiana S.A. $2015^{\circ}$

Recibido el 01 de mayo de 2015; aceptado el 10 de junio de 2015 
Introduction: candidemia is a serious disease with high morbidity and mortality whose treatment does not always lead to a cure. Candida species distribution and antifungal susceptibility varies by geographic area, even in health centers in a given region. Objective: To establish Candida species distribution and in vitro susceptibility to different antifungal agents. Material and methods: A descriptive, prospective, and cross-sectional study of candidemia cases was performed at a health institution in Valledupar, Colombia between November 2013 and May 2014. The Minimum Inhibitory Concentrations (MIC) were determined following the M27-A3-S4 standard protocol. Results: 40 isolates of Candida spp. obtained from blood (97.5\%) and bone marrow (2.5\%) were analyzed. Candida tropicalis, 15 (37.5\%), Candida albicans Complex, 13 (32.5\%), Candida glabrata Complex, five ( $12.5 \%)$, Candida guilliermondii, three (7.5\%), Candida parapsilosis Complex 3 (7.5\%) and Candida krusei, one (2.5\%) were isolated. No resistance of isolates to amphotericin B and voriconazole was observed; in contrast, one (6.6\%) isolate of Candida tropicalis and one (33.3\%) of Candida parapsilosis Complex showed resistance to fluconazole and a single isolate of Candida krusei and one (20\%) of Candida glabrata Complex to caspofungin. Conclusions: The local epidemiology of causative yeasts for candidemia showed higher prevalence of non-albicans species with resistance to antifungal agents evaluated, which is relevant to the election and establishment of more effective treatment.

Key words: Candida, candidemia, antimicrobial susceptibility tests, antifungal agents

Vides-Peña CA, Bolaño-Ardila ND, Vides-Peña MV, Córdoba SB. Epidemiología de la candidemia y sensibilidad in vitro frente a azoles, anfotericina B y caspofungina en una institución de salud de Valledupar, Colombia. Medicina \& Laboratorio 20 I 5; 21: 255-266.

E estudio epidemiológico de las entidades fúngicas constituye un elemento importante en la dinámica de su diagnóstico y tratamiento, especialmente en pacientes que poseen disfunciones del sistema inmune $u$ otros factores de riesgo asociados a la ocurrencia de procesos infecciosos invasivos [ 1 ]. Candida spp. se presenta como el género levaduriforme más frecuentemente implicado en la etiología de estos episodios en las Unidades de Cuidados Intensivos $(\cup C I)$, en donde se asocia con una mortalidad que puede alcanzar cifras cercanas al 80\% [2,3].

Los aspectos epidemiológicos de la candidemia han sido más ampliamente estudiados en los Estados Unidos y Europa que en América Latina [4]. No obstante, los datos derivados del primer estudio multicéntrico sobre candidemia desarrollado en varios países de Latinoamérica entre 2008 y 2010 mostraron una alta incidencia, alta proporción de niños afectados (no solo neonatos) y una distribución de especies marcada por una mayor frecuencia de Candida tropicalis, Candida parapsilosis y Candida guilliermondii, contrastadas con frecuencias menores para aislados de Candida glabrata [5]. Investigaciones realizadas en el mismo contexto han demostrado que el comportamiento de la respuesta a los antifúngicos y la distribución que muestran las especies del género Candida han variado de manera importante de acuerdo a la ubicación geográfica, el servicio de hospitalización y los factores de riesgo de los pacientes [6].

Considerando estos importantes aspectos y para aportar herramientas epidemiológicas útiles en el diseño de los esquemas terapéuticos empíricos usados en el tratamiento de la candidiasis invasora en los centros asistenciales, en el presente estudio se analizó la distribución de especies y el perfil de sensibilidad a fluconazol, voriconazol, anfotericina B y caspofungina de 40 aislados clínicos del género Candida, obtenidos a partir de muestras de sangre y médula ósea 
de pacientes hospitalizados entre noviembre de 2013 y mayo de 2014 en una institución de salud en Valledupar, Colombia.

\section{Materiales y métodos}

\section{Diseño y población de estudio}

Se realizó un estudio observacional descriptivo, prospectivo y transversal en el que se definió como población de estudio el total de aislados clínicos no repetitivos del género Candida provenientes de muestras obtenidas de sitios estériles en pacientes atendidos entre el 01 de noviembre de 2013 y el 0 I de mayo de 2014 en una institución de salud de la ciudad de Valledupar, Cesar, Colombia.

Durante el tiempo de intervención el centro asistencial prestó, con un porcentaje de ocupación aproximado al $98 \%$, servicios de baja, mediana y alta complejidad, incluyendo servicios de mediana complejidad con algunas actividades de alta complejidad; contó con 228 camas distribuidas en los servicios de Hospitalización (HOSP), Ginecología y Obstetricia (GOB), Unidad de Cuidados Intermedios Neonatales (UCINN), Unidad de Cuidados Intensivos Neonatales (UCIN), Unidad de Cuidados Intermedios Adultos (UCINA) y Unidad de Cuidados Intensivos Adultos (UCIA), y tuvo promedios generales de días de estancia y giro de cama-mes cercanos a tres y 12 , respectivamente.

Se excluyeron de la investigación los aislados que manifestaron dificultades para el crecimiento durante su mantenimiento rutinario o que no fueron caracterizadas como Candida spp. tras su reconfirmación fenotípica. Las levaduras recolectadas se acompañaron de información demográfica de los pacientes (edad, sexo y servicio de hospitalización) obtenida previa autorización del centro asistencial.

\section{Caracterización y pruebas de sensibilidad de Candida spp.}

Las levaduras fueron aisladas de hemocultivos y mielocultivos procesados por el método automatizado BacT/ALERT ${ }^{\circledR}$ (BioMérieux, Marcy-l'Étoile, Francia). La caracterización fenotípica de las especies fúngicas se realizó mediante el sistema MicroScan ${ }^{\circledR}$ Rapid Yeast Identification (RYI) ${ }^{\circledR}$ (Siemens, Múnich, Alemania), complementado con estudios morfofisiológicos sugeridos por protocolos estandarizados [7], entre los que se incluyeron: a) evaluación macro y micromorfológica de los aislados usando CHROMagarTM Candida (CHROMagar Company Ltda., París, Francia), agar Yeast Mould (YM) ${ }^{\circledR}$ (Oxoid, Hampshire, Reino Unido) y caldo Extracto de Malta ${ }^{\circledR}$ (Merck Millipore, Darmstadt, Alemania) al 5\%, b) crecimiento a diferentes temperaturas, c) zimogramas para la evaluación de la fermentación de los carbohidratos: glucosa, galactosa, sacarosa, maltosa, lactosa, trehalosa y rafinosa, y d) auxonogramas para la asimilación de los compuestos carbonados: glucosa, galactosa, L-sorbosa, sacarosa, maltosa, celobiosa, trehalosa, lactosa, melibiosa, D-xilosa, L-arabinosa, D-ribosa, D-manitol, inositol, L-rhamnosa, eritritol y citrato, y para los nitrogenados: nitrato de potasio y peptona.

La evaluación de la actividad in vitro del fluconazol y el voriconazol (Pfizer, Nueva York, Estados Unidos), la anfotericina B (Sigma-Aldrich, San Luis, Misuri, Estados Unidos) y la caspofungina (Merck Millipore) se realizó mediante la técnica de microdilución en caldo utilizando el medio sintético RPMI- 640 con glutamina, sin bicarbonato, con rojo fenol como indicador de pH (SigmaAldrich) y glucosa al 0,2\%. Los rangos de concentraciones probados para las soluciones antifúngicas fueron de $0,015 \mu \mathrm{g} / \mathrm{mL}$ a $16 \mu \mathrm{g} / \mathrm{mL}$ para la anfotericina $\mathrm{B}$, de $0,13 \mu \mathrm{g} / \mathrm{mL}$ a $128 \mu \mathrm{g} / \mathrm{mL}$ para 
el fluconazol y de $0,03 \mu \mathrm{g} / \mathrm{mL}$ a $16 \mu \mathrm{g} / \mathrm{mL}$ para la caspofungina y el voriconazol. Las soluciones madre se prepararon en dimetilsulfóxido (DMSO) (Sigma-Aldrich).

La preparación de los inóculos, el tiempo y la temperatura de incubación, al igual que la lectura y la interpretación de los resultados, se definieron según lo dispuesto en el protocolo estándar M27-A3-S4 del Instituto de Estándares Clínicos y de Laboratorio (CLSl; del inglés, Clinical and Laboratory Standards Institute) [8,9]. Se consideró como la Concentración Inhibitoria Mínima (CIM) aquella que provocó una disminución en el desarrollo levaduriforme de un $95 \%$ en el caso de la anfotericina B y del $50 \%$ para los azoles y la caspofungina, en comparación con la obtenida en el control de crecimiento. Los puntos de corte utilizados para la determinación de las concentraciones inhibitorias mínimas se muestran en la tabla I, excepto para la anfotericina B, con la cual se adoptaron los sugeridos según las concentraciones inhibitorias mínimas poblacionales de los aislados [8- 10]. Además, se calcularon los valores del rango, la Concentración Inhibitoria Mínima $50\left(\mathrm{CIM}_{50}\right)$, la Concentración Inhibitoria Mínima $90\left(\mathrm{ClM}_{90}\right)$ y el porcentaje de resistencia para cada aislado. En todas las pruebas se incluyeron las cepas control de Candida parapsilosis ATCC ${ }^{\circledR} 22019$ y Candida krusei ATCC $^{\circledR} 6258$.

\section{Análisis de datos}

Se realizó un análisis descriptivo usando los software Microsoft Exce ${ }^{\circledR} 2007$ (Microsoft Corporation, Washington, Estados Unidos) y SPSS ${ }^{\circledR}$ versión I5 (IBM, Armonk, Nueva York, Estados Unidos).

\section{Aspectos éticos}

Se cumplieron las disposiciones de la Resolución 8430 de 1993 del Ministerio de Salud de la República de Colombia [ I I ] y demás reglamentaciones bioéticas internacionales vigentes. La investigación se consideró sin riesgo y no fue necesaria la solicitud de consentimiento informado en ninguna de sus fases. Las levaduras y la información demográfica derivadas de los pacientes (edad, sexo y servicio de hospitalización) se recolectaron y usaron con la debida autorización del centro asistencial, garantizándose siempre el derecho de confidencialidad de la información obtenida y la protección de su buen nombre. Se respetaron en todas las etapas de trabajo los 

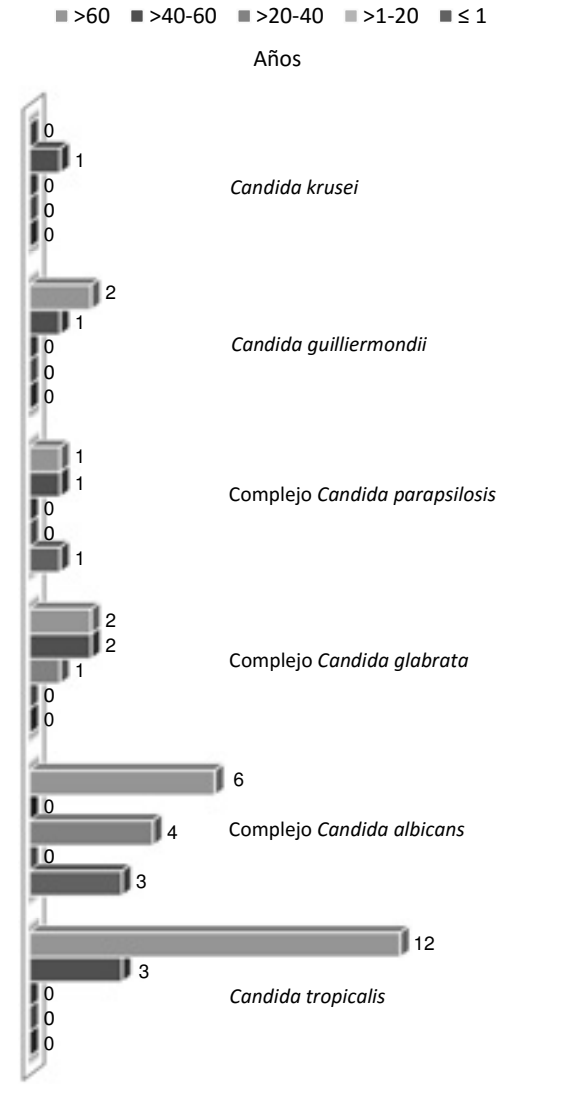

Figural. Distribución de especies de Candida según grupo etario $\because \mathrm{HOSP}=\mathrm{UCIN} \backsim \mathrm{UCIA}$

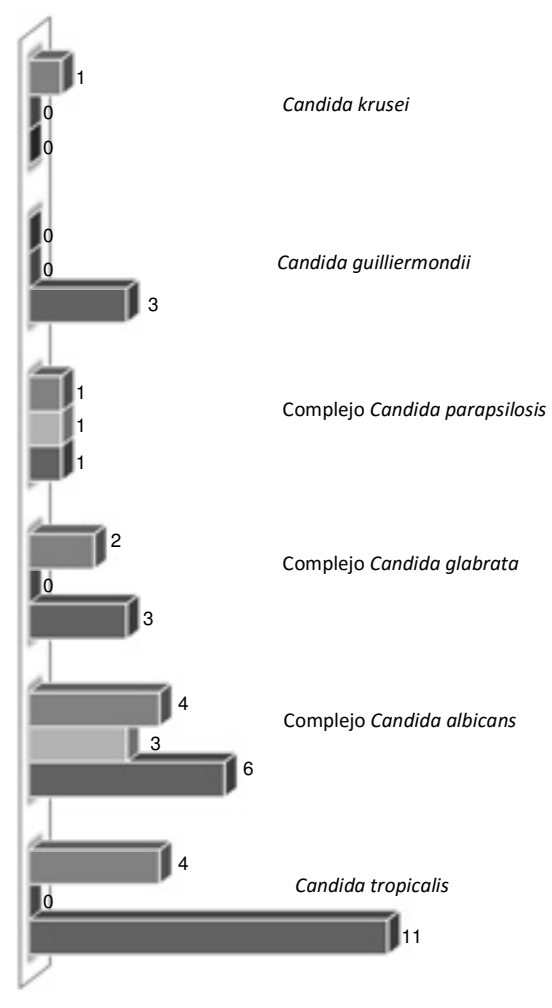

Figura 2. Distribución de especies de Candida según servicio de atención. HOSP: Hospitalización; UCIN: Unidad de Cuidados Intensivos Neonatales; UCIA: Unidad de Cuidados Intensivos para Adultos

estándares universales de bioseguridad para la recolección, procesamiento, mantenimiento y transporte de los microorganismos.

\section{Resultados}

En el período de estudio se recolectaron un total de 43 aislados clínicos no repetitivos del género Candida, de los cuales se excluyeron dos debido a dificultades para el crecimiento durante su mantenimiento y uno que no se caracterizó como Candida spp. tras su reconfirmación fenotípica, para un total de 40 aislados. No se evidenció la coexistencia de especies ni se encontraron diferencias importantes en la frecuencia de aislados clínicos entre los pacientes masculinos $(n=21)$ y los femeninos $(n=19)$. El grupo etario para el que se reportó mayor número de casos fue el conformado por los mayores de 60 años con 57,5\% (23/40) de los aislados (véase figura I y tabla 2). De los 40 aislados clínicos de Candida spp. incluidos, el 97,5\% (39/40) fue obtenido a partir de muestras de sangre y el 2,5\% (1/40) de médula ósea. El 67,5\% (27/40) de los aislados se clasificaron como especies no-albicans: el 37,5\% (I5/40) para Candida tropicalis, el I2,5\% (5/40) para el Complejo Candida glabrata, el 7,5\% (3/40) tanto para Candida guilliermondii como para el Complejo Candida parapsilosis y el 2,5\% (I/40) para Candida krusei. El 32,5\% ( 13/40) de aislados restantes se caracterizaron como pertenecientes al Complejo Candida albicans. 


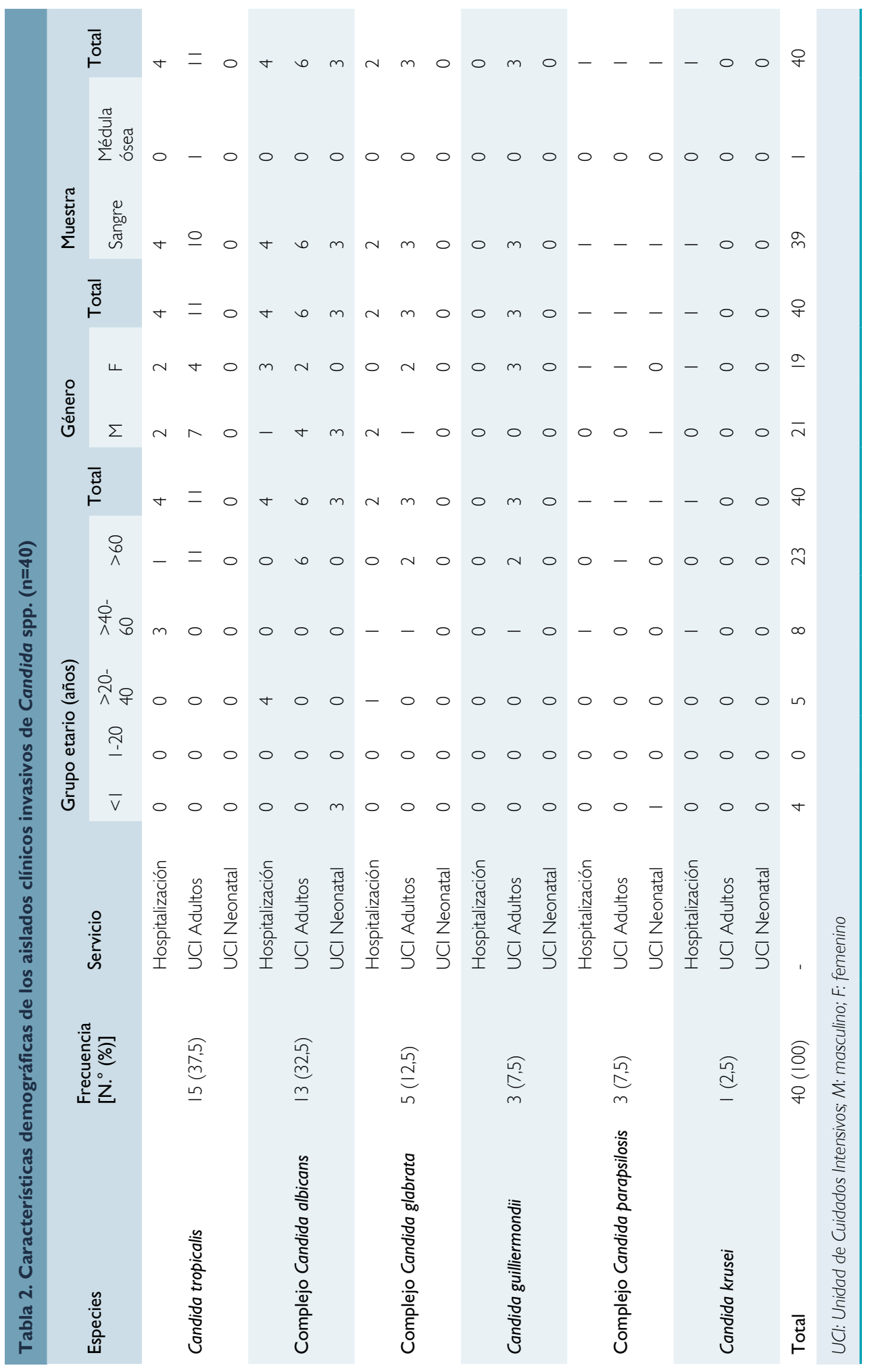


De todos los casos de candidemia incluidos, el 60\% (24/40) se registraron en la Unidad de Cuidados Intensivos para Adultos (UCIA), con el 27,5\% ( 1 1/40) de los aislados clínicos caracterizados como Candida tropicalis, el 30\% (12/40) provinieron del Servicio de Hospitalización (HOSP), con el 10\% (4/40) de los aislados clasificados tanto para Candida tropicalis como para el Complejo Candida albicans, y en menor proporción de las Unidades de Cuidados Intensivos Neonatales (UCIN) con el 10\% (4/40) de los aislados, 7,5\% (3/40) identificados como del Complejo Candida albicans (véase figura 2).

Los valores de las Concentraciones Inhibitorias Mínimas (CIM) obtenidas para los antifúngicos ensayados en los aislados de Candida spp. se muestran en la tabla 3. Todos los aislados fueron sensibles a la anfotericina $B(C \mid M \leq 1,0 \mu \mathrm{g} / \mathrm{mL})$. En los aislados evaluados con voriconazol no se observó resistencia (rango de $\mathrm{CIM}=0,015 \mu \mathrm{g} / \mathrm{mL}$ a $0,5 \mu \mathrm{g} / \mathrm{mL}$ ), pero se obtuvieron resultados de sensibilidad dependiente de la dosis (CIM=0,25 $\mathrm{mg} / \mathrm{mL})$ en el 15,4\% (2/13) de los aislados del Complejo Candida albicans. El 6,6\% (I/I5) de los aislados de Candida tropicalis y el 33,3\% ( $1 / 3)$ de los del Complejo Candida parapsilosis resultaron resistentes al fluconazol (CIM=8 $\mu \mathrm{g} /$ $\mathrm{mL}$ y $32 \mu \mathrm{g} / \mathrm{mL}$, respectivamente); en el 15,4\% (2/13) de los aislados del Complejo Candida albicans y en el 6,6\% (I/I5) de Candida tropicalis se observó sensibilidad dependiente de la dosis a este mismo antifúngico (CIM=4 $\mu \mathrm{g} / \mathrm{mL})$. El aislado caracterizado como Candida krusei, así como el 20\% (I/5) de los pertenecientes al Complejo Candida glabrata mostraron resistencia a la caspofungina (CIM $\geq 1,0 \mu \mathrm{g} / \mathrm{mL} y \geq 0,5 \mu \mathrm{g} / \mathrm{mL}$, respectivamente). Los resultados de categoría intermedia para esta equinocandina se observaron también en el 40\% (2/5) de los aislados del Complejo Candida glabrata y el 13,3\% (2/15) de Candida tropicalis.

\section{Discusión}

En este trabajo, la Unidad de Cuidados Intensivos para Adultos (UCIA) fue el servicio en el que se presentaron episodios de candidemia con mayor frecuencia, particularmente, en el grupo de pacientes mayores de 60 años. Estos datos corresponden con los expresados por otros autores en trabajos epidemiológicos sobre candidemia, en donde la estancia, especialmente si es prolongada, en estas unidades y la disminución de la actividad inmune asociada a la senectud o a las enfermedades de base, son factores predisponentes bien conocidos para la ocurrencia de cuadros fúngicos invasivos $[5,12]$. Sin embargo, los hallazgos de investigaciones relacionadas $[5,13]$ demuestran la necesidad de implementar también en las Unidades de Cuidados Intensivos Neonatales (UCIN) y demás servicios hospitalarios protocolos efectivos de profilaxis y control de infecciones que contribuyan a la disminución de las candidemias en la población pediátrica, inclusive si la frecuencia de estas es baja.

Respecto a las especies aisladas, las de Candida tropicalis y el Complejo Candida albicans fueron las que se encontraron con mayor frecuencia. Estos datos son comparables a los reportados en Colombia en estudios previos realizados por Villar y colaboradores (2004) [14], en el que se mostró una frecuencia de aislados del 45\% para Candida albicans y Candida tropicalis, y del I\% para Candida glabrata y Candida parapsilosis, y por Cortés y colaboradores (2013) [15], quienes a partir de un estudio multicéntrico en el que participaron siete hospitales de la ciudad de Bogotá indicaron que, en su orden, Candida albicans, Candida tropicalis y Candida parapsilosis fueron las especies más frecuentemente aisladas. Si bien la frecuencia de los aislados no es idéntica, en el presente trabajo sí se encontró una aproximación cercana a la distribución de especies expuesta por estos investigadores. 
Tabla 3. Sensibilidad in vitro de Candida spp. a cuatro agentes antifúngicos

\begin{tabular}{|c|c|c|c|c|c|}
\hline Antifúngico & Especies & $\mathrm{CIM}_{50}(\mu \mathrm{g} / \mathrm{mL})$ & $\mathrm{CIM}_{90}(\mu \mathrm{g} / \mathrm{mL})$ & Rango & $\begin{array}{l}\text { Resistencia } \\
{\left[\mathrm{N} .^{\circ}(\%)\right]}\end{array}$ \\
\hline \multirow{6}{*}{ Anfotericina B } & Candida tropicalis $(n=15)$ & 0,5 & 1,0 & $0,06-1,0$ & $0(0)$ \\
\hline & $\begin{array}{l}\text { Complejo Candida albicans } \\
(n=\mid 3)\end{array}$ & 0,25 & 0,5 & $0,13-1,0$ & $0(0)$ \\
\hline & $\begin{array}{l}\text { Complejo Candida glabrata } \\
(n=5)\end{array}$ & 1,0 & 1,0 & $0,25-1,0$ & $0(0)$ \\
\hline & Candida guilliermondii $(n=3)$ & NA & NA & $0,5-1,0$ & $0(0)$ \\
\hline & $\begin{array}{l}\text { Complejo Candida parapsilosis } \\
(n=3)\end{array}$ & NA & NA & $0,13-1,0$ & $0(0)$ \\
\hline & Candida krusei $(\mathrm{n}=\mathrm{l})$ & NA & NA & 0,25 & $0(0)$ \\
\hline \multirow{6}{*}{ Fluconazol } & Candida tropicalis $(n=15)$ & 1,0 & 4,0 & $0,25-8,0$ & I $(6,6)$ \\
\hline & $\begin{array}{l}\text { Complejo Candida albicans } \\
(n=13)\end{array}$ & 0,5 & 4,0 & $0,13-4,0$ & $0(0)$ \\
\hline & $\begin{array}{l}\text { Complejo Candida glabrata } \\
(n=5)\end{array}$ & 2,0 & 8,0 & $0,5-16,0$ & $0(0)$ \\
\hline & Candida guilliermondii $(n=3)$ & NA & NA & $0,5-8,0$ & NE \\
\hline & $\begin{array}{l}\text { Complejo Candida parapsilosis } \\
(n=3)\end{array}$ & NA & NA & $0,5-32,0$ & I $(33,3)$ \\
\hline & Candida krusei $(n=1)$ & NA & NA & 32 & I $(100)^{\ddagger}$ \\
\hline \multirow{6}{*}{ Voriconazol } & Candida tropicalis $(n=15)$ & 0,06 & 0,06 & $0,015-0,06$ & $0(0)$ \\
\hline & $\begin{array}{l}\text { Complejo Candida albicans } \\
(n=\mid 3)\end{array}$ & 0,03 & 0,25 & $0,0 \mid 5-0,25$ & $0(0)$ \\
\hline & $\begin{array}{l}\text { Complejo Candida glabrata } \\
(\mathrm{n}=5)\end{array}$ & 0,06 & 0,06 & $0,03-0,5$ & NE \\
\hline & Candida guilliermondii $(n=3)$ & NA & NA & $0,015-0,06$ & NE \\
\hline & $\begin{array}{l}\text { Complejo Candida parapsilosis } \\
(n=3)\end{array}$ & NA & NA & 0,06 & $0(0)$ \\
\hline & Candida krusei $(\mathrm{n}=\mathrm{I})$ & NA & NA & 0,13 & $0(0)$ \\
\hline \multirow{6}{*}{ Caspofungina } & Candida tropicalis $(n=15)$ & 0,13 & 0,5 & $0,015-0,5$ & $0(0)$ \\
\hline & $\begin{array}{l}\text { Complejo Candida albicans } \\
(n=\mid 3)\end{array}$ & 0,03 & 0,06 & $0,015-0,06$ & $0(0)$ \\
\hline & $\begin{array}{l}\text { Complejo Candida glabrata } \\
(n=5)\end{array}$ & 0,25 & 0,25 & $0,13-0,5$ & I $(20,0)$ \\
\hline & Candida guilliermondii $(n=3)$ & NA & NA & $0,5-1,0$ & $0(0)$ \\
\hline & $\begin{array}{l}\text { Complejo Candida parapsilosis } \\
(n=3)\end{array}$ & NA & NA & $0,06-1,0$ & $0(0)$ \\
\hline & Candida krusei $(n=1)$ & NA & NA & 1,0 & I $(100,0)$ \\
\hline
\end{tabular}

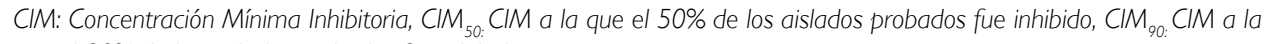
que el $90 \%$ de los aislados probados fue inhibido.

NE: No Establecido, NA: No Aplica (el número de aislados se consideró bajo para los cálculos de $C_{1} M_{50}$ y $C_{1} M_{90}$ ).

*: Resistencia intrínseca

Asimismo, los resultados comunicados por Nucci y colaboradores (20 I3) [5] revelaron un porcentaje frecuente de aislados de Candida no-albicans en 20 centros asistenciales de siete países latinoamericanos, siendo en Brasil, Chile, Argentina y Ecuador, los aislamientos del Complejo Candida parapsilosis y Candida tropicalis los que se obtuvieron en segundo y tercer porcentaje de frecuencia, respectivamente, luego de los del Complejo Candida albicans. Para Colombia y Venezuela, el Complejo Candida parapsilosis fue referido como el más frecuente, seguido del Complejo Candida albicans y Candida tropi- 
calis, mientras que en Honduras Candida tropicalis ocupó la segunda frecuencia de aislamiento, después del Complejo Candida albicans, pero antecediendo al Complejo Candida parapsilosis.

En Argentina, Santos y colaboradores (2010) [16], al evaluar la distribución y perfil de sensibilidad in vitro según el estándar del CLSI a la anfotericina B, el fluconazol y el itraconazol de levaduras de interés médico involucradas en 49 episodios de fungemias, indicaron que Candida parapsilosis fue la especie más frecuente con el 32,6\%, seguida de Candida albicans con el 26,5\% y Candida tropicalis con el 24,5\%. En este mismo país, Comistein y colaboradores (2013) [17] demostraron que a partir de muestras provenientes de sitios estériles el mayor número de aislamientos fue para las especies no-albicans, siendo, en orden de aparición, Candida tropicalis, Candida parapsilosis, Candida glabrata y Candida knusei las más frecuentes. En Venezuela, García y Hemández (2013) [ 18], informaron que durante su estudio el porcentaje de aislamiento fue igualmente mayor para las especies no-albicans, siendo Candida tropicalis la más frecuente a partir de muestras de orina y puntas de catéter, seguida de Candida glabrata, Candida parapsilosis, Candida guilliemondii y Candida kefyr.

De igual modo, el estudio multicéntrico de la red FUNGEMYCA, realizado en España por Montejo y colaboradores (201 I) [19], en donde se estudiaron casos de fungemia en 43 hospitales durante un período de 13 meses, entre 2009 y 20 I0, expuso una distribución de frecuencia similar de las principales especies del género Candida: 43\% para el Complejo Candida albicans y 30\% para el Complejo Candida parapsilosis. No obstante, se estableció un porcentaje de aislados del I 1\% para el Complejo Candida glabrata, lo que demuestra que la distribución de algunas especies difiere geográficamente. En tanto, se ha descrito que Candida glabrata es especialmente prevalente en el norte de Europa y Norteamérica, mientras que Candida parapsilosis es más frecuente en el sur de Europa y América Latina [20].

En todos los ámbitos es destacable que las llamadas especies no-albicans han aumentado globalmente respecto al pasado [21-25], cuando debido al menor número de trasplantes, procedimientos invasivos, colocación de dispositivos protésicos, pacientes infectados con el virus de la inmunodeficiencia humana $(\mathrm{VIH})$, padecimientos oncológicos, dismetabolias, migraciones humanas, e incluso, a las limitaciones técnicas que existían en los laboratorios de microbiología clínica para la caracterización fenotípica de levaduras, la epidemiología proponía a Candida albicans como la especie de mayor importancia médica [26,27]. En la actualidad, se ha propuesto también que el uso temprano o profiláctico de antifúngicos de la familia de los azoles, específicamente fluconazol, contribuye al cambio en el patrón epidemiológico, favoreciendo el aumento de las especies no-albicans [15].

En cuanto a la sensibilidad antifúngica, si bien se han descrito en la literatura bajas tasas de resistencia a la anfotericina B en especies de Candida, especialmente en las no-albicans [23], los resultados de este estudio mostraron sensibilidad a este fármaco, pues ningún aislado exhibió una concentración inhibitoria mínima mayor al punto de corte (> $>$ mg/mL), lo que sugiere, que tal como lo ha propuesto la "Guía de práctica clínica para el manejo de la candidiasis" de la Sociedad de Enfermedades Infecciosas de América (IDSA; del inglés, The Infectious Diseases Society of America) [28-30], el fármaco puede considerarse una terapia altemativa efectiva para el tratamiento de las candidemias en la mayoría de los pacientes e instituciones de salud. No obstante, es importante indicar que actualmente el protocolo estándar vigente del Instituto de Estándares Clínicos y de Laboratorio (CLSI), el M27-A3-S4, no ha propuesto puntos de corte clínicos para la evaluación de la sensibilidad a este antifúngico y, por tanto, los resultados obtenidos por esta metodología son interpretados de acuerdo a los puntos de corte epidemiológicos derivados de las concentraciones inhibitorias mínimas poblacionales que son, a su vez, similares a los propuestos por el protocolo estándar E.Def 7.2 del Comité Europeo de Pruebas de Sensibilidad Antimicrobiana (EUCAST; del inglés, European Committee on Antimicrobial Susceptibility Testing) [3I] para algunas especies del género Candida. 
Referente a los perfiles de sensibilidad de los azoles, para el voriconazol el I5,4\% de los aislados del Complejo Candida albicans presentó una sensibilidad dependiente de la dosis (CIM=0,25 $\mu \mathrm{g} / \mathrm{mL}$ ), de forma similar a lo obtenido en el estudio de Cuenca-Estrella y colaboradores (20 I I) [32] aun usando los puntos de corte propuestos por el EUCAST. En relación con esto, Pfaller y colaboradores (2010) [33] referenciaron en su estudio la concordancia de los protocolos estándares del CLSI y el EUCAST, e indicaron una concordancia esencial (concordancia dentro de, más/menos una dilución entre ambos) que va desde el 96,9\% para el voriconazol hasta el 98,6\% para el fluconazol, al ensayar especies de Candida.

Para el fluconazol se evidenció la resistencia en el aislado de Candida krusei; especie reconocida como resistente intrínseca a este fármaco. Los porcentajes de resistencia (CIM $\geq 8 \mu \mathrm{g} / \mathrm{mL})$ observados en los aislados de Candida tropicalis y del Complejo Candida parapsilosis no se correlacionaron de manera uniforme con los reportados para estas especies, lo que demuestra que realmente hay una variación de los perfiles de sensibilidad de acuerdo a la ubicación geográfica e institución de salud de la que se obtuvieron los aislados clínicos. En el mismo sentido, se ha descrito con preocupación un incremento en el número de aislados de Candida albicans resistentes, puesto que solía ser Candida glabrata la especie que con mayor frecuencia exhibía este perfil [5,34,35]. Al respecto, en el I5,4\% de los aislados del Complejo Candida albicans y el 6,6\% de Candida tropicalis se observó una sensibilidad dependiente de la dosis a este antifúngico $(\mathrm{CIM}=4 \mu \mathrm{g} / \mathrm{mL})$. Todo ello pudiera explicarse desde varias perspectivas que incluyen la posibilidad de resistencia emergente mediada por mecanismos conjuntos descritos en las levaduras (p. ej. bombas de eflujo, cambios estructurales en el sitio blanco o sobreexpresión de la enzima Erg I l p, mutaciones en el gen ERG3, defectos genéticos o respiratorios en las levaduras) a causa de la presión selectiva inducida por el uso previo o descontrolado de los medicamentos, que origina usualmente resistencia cruzada para el fluconazol y el voriconazol [2], y la capacidad que poseen la mayoría de especies de Candida para producir biopelículas, en las cuales los azoles no tienen mayor actividad [36].

Para la caspofungina el Complejo Candida glabrata mostró una resistencia en el $20 \%$ de los aislados, al igual que el único aislado de Candida krusei obtenido (CIM $\geq 0,5 \mu \mathrm{g} / \mathrm{mL}$ y $\mathrm{CIM} \geq 1,0 \mu \mathrm{g} / \mathrm{mL}$, respectivamente). Los resultados que al respecto han comunicado otros estudios son diversos, algunos con mayores porcentajes de resistencia y otros con frecuencias menores; no obstante, se ha postulado que Candida glabrata y otras especies del género Candida pueden desarrollar con cierta facilidad resistencia a las equinocandinas tras mutaciones del gen FKS $[2,37,38]$, por lo que pudiera proponerse al aislado resistente del Complejo Candida glabrata como candidato para determinar una mutación a nivel de este gen. En referencia a ello, las investigaciones de Pfaller y colaboradores (20 I4) [39] y otros autores [40,4 I] han propuesto la posibilidad de usar la micafungina como predictora del grado de sensibilidad de las especies de Candida frente a la caspofungina, lo que sería útil si se tiene en cuenta que el estándar E.Def 7.2 del EUCAST no posee puntos de corte clínicos para la interpretación de la caspofungina, debido a las significativas variaciones interlaboratorios que se han evidenciado en los resultados de las pruebas.

Es claro que las especies no-albicans han mostrado un incremento significativo en la etiología de las candidiasis invasivas [3,42] y su importancia como patógenos emergentes radica no solo en la distribución epidemiológica en relación a la geografía, tipos de pacientes que infectan y cuadros que causan, sino también en las variaciones que suelen demostrar en la respuesta al tratamiento antifúngico. Por tal razón, incluso con las limitaciones técnicas que existen en algunos laboratorios de microbiología clínica, las diferencias entre los protocolos estándares propuestos por los diferentes comités y las discrepancias que puedan observarse entre los resultados obtenidos en los antifungigramas y la respuesta clínica que se evidencia en el seguimiento de los pacientes, sigue siendo necesario caracterizar la identidad y el perfil de sensibilidad de las levaduras [43]. 
Es imperioso que las futuras investigaciones orientadas a fortalecer la epidemiología local y global de las candidemias incluyan un número mayor de aislados clínicos, vinculen a más instituciones de salud y consideren un seguimiento clínico riguroso para la obtención de información referente a la farmacoterapia y evolución de los pacientes, buscando así generar panoramas epidemiológicos más completos que puedan apoyar efectivamente el establecimiento de puntos de corte epidemiológicos y clínicos para la formulación de guías terapéuticas consonantes con la circulación y perfil de sensibilidad de las especies levaduriformes.

\section{Agradecimientos}

A la Vicerrectoría de Investigación y Extensión de la Universidad Popular del Cesar (sede central), por la financiación de esta investigación en el marco de la Convocatoria Interna para la Financiación de Proyectos de Investigación a Grupos de Investigación avalados en la Plataforma ScienTI de Colciencias, aprobada por el Comité Central de Investigación y ejecutada según lo dispuesto en el Convenio de Desempeño N. ${ }^{\circ}$ I 12 suscrito entre la universidad y la investigadora principal.

A Graciela Davel, Guillermina Isla, Walter Vivot, Wanda Szuzs, Matías Vivot, Omar Murisengo y Soraya Morales, por su infinita hospitalidad y excelente asistencia técnica durante la estancia en los Laboratorios de Levaduras y Antifúngicos del Departamento de Micología, Instituto Nacional de Enfermedades Infecciosas (INEI-ANLIS) "Dr. Carlos G. Malbrán" de la Ciudad Autónoma de Buenos Aires, Argentina, y a Catalina de Bedout de la Corporación para Investigaciones Biológicas (CIB), Medellín, Colombia, por sus valiosos aportes.

\section{Bibliografía}

I. Akeme Yamamoto AC, de Paula CR, Dias LB, Tadano T, Martins ER, Amadio JV, et al. Epidemiological and clinical characteristics of nosocomial candidiasis in university hospitals in Cuiaba-Mato Grosso, Brazil. Rev Iberoam Micol 20 I2; 29: $164-168$.

2. Maubon D, Garnaud C, Calandra T, Sanglard D, Cornet M. Resistance of Candida spp. to antifungal drugs in the ICU: where are we now? Intensive Care Med 20 I4; 40: I24 I - 255.

3. de Bedout C, Gómez BL. Candida y candidiasis invasora: un reto continuo para su diagnóstico temprano. Infectio 20 I0; |4: s|59-s|7|.

4. Colombo AL, Nucci M, Park BJ, Nouer SA, ArthingtonSkaggs B, da Matta DA, et al. Epidemiology of candidemia in Brazil: a nationwide sentinel surveillance of candidemia in eleven medical centers. J Clin Microbiol 2006; 44: 2816-2823.

5. Nucci M, Queiroz-Telles F, Alvarado-Matute T, Tiraboschi IN, Cortes J, Zurita J, et al. Epidemiology of candidemia in Latin America: a laboratory-based survey. PLoS One 20 I3; 8: e59373.

6. Gómez Quintero $\mathrm{CH}$. Resistencia de levaduras del género Candida al fluconazol. Infectio 20 10; |4: s |72-s 180.

7. Kurtzman C, Fell JW, Boekhout T. The Yeasts: A taxonomic Study (ed 5a). Londres, Reino Unido: Elsevier Science; 201 I.

8. Clinical and Laboratory Standards Institute (CLSI). Reference Method for Broth Dilution Antifungal Susceptibility Testing of Yeasts; Approved Standard-Third Edition. CLSI document M27-A3. Pensilvania, Estados Unidos: Clinical and Laboratory Standards Institute; 2008.
9. Clinical and Laboratory Standards Institute (CLSI). Reference Method for Broth Dilution Antifungal Susceptibility Testing of Yeasts; Fourth Informational Supplement. CLSI document M27-S4. Pensilvania, Estados Unidos: Clinical and Laboratory Standards Institute; 2012.

10. Córdoba S, Vivot W, Szusz W. Curso a distancia y Taller "Determinación de la resistencia a antifúngicos en el laboratorio". Caba, Argentina: Departamento Micología INEI, ANLIS "Dr. Carlos G. Malbran". 2013.

II. Republica de Colombia, Ministerio de Salud. Resolución No 008430. 1993. Disponible: https:/unww.invima. gov.co/images/pdf/medicamentos/resoluciones/etica_ res_8430_1993.pdf. Consultado: jul 2013.

12. Almirante B, Rodriguez D, Park BJ, Cuenca-Estrella M, Planes AM, Almela M, et al. Epidemiology and predictors of mortality in cases of Candida bloodstream infection: results from population-based surveillance, barcelona, Spain, from 2002 to 2003. J Clin Microbiol 2005; 43: I829- 1835.

13. Coria P. Fungemias asociadas a catéter por Candida sp. en neonatología. Rev Chil Infectol 2012; 29: 479-479.

14. Villar Centeno LÁ, Díaz Quijano FA, Céspedes Jl, Torres A, De Bedout C. Prevalencia y patrones de sensibilidad al Fluconazol de las especies de Candida aisladas en pacientes de Unidades de Cuidado Intensivo de Bucaramanga, Colombia. Infectio 2004; 8: 185-193.

15. Cortés JA, Jaimes JA, Leal AL. Incidencia y prevalencia de candidemia en pacientes críticamente enfermos en Colom- 
bia. Rev Chil Infectol 2013; 30: 599-604

16. Santos PE, Córdoba S, Carrillo-Muñoz A, Rodero L, Rubeglio E, Soria M. Epidemiología de las fungemias en un hospital pediátrico de alta complejidad. Rev Iberoam Micol 2010; 27: 200-202.

17. Cornistein W, Mora A, Orellana N, Capparelli F, del Castillo M. Candida: epidemiología y factores de riesgo para especies no albicans. Enferm Infecc Microbiol Clín 20 13; 31: 380-384

18. García YS, Hernández Valles R. Aislamiento y susceptibilidad a fluconazol y voriconazol de especies de Candida provenientes de pacientes recluidos en la Unidad de Cuidados Intensivos, Hospital Universitario de Coro, estado Falcón, Venezuela. Rev Soc Ven Microbiol 2013; 33: I40- 146.

19. Montejo M, Quindós G. Evidencias científicas que respaldan el uso de micafungina en el tratamiento de la candidiasis invasora. Enferm Infecc Microbiol Clin 201 I; 29: 15-22.

20. Cantona E, García-Rodríguez J, Martín-Mazuelos E, Pemána J, Guinea J. Métodos microbiológicos para el diagnóstico, manejo y estudio de la infección fúngica invasora. Enferm Infecc Microbiol Clin 2014; 32: 375-379.

21. Khan Z, Ahmad S, Joseph L, Chandy R. Candida dubliniensis: an appraisal of its clinical significance as a bloodstream pathogen. PLoS One 2012; 7: e32952.

22. Yu N, Kim HR, Lee MK. The first Korean case of candidemia due to Candida dubliniensis. Ann Lab Med 2012; 32: 225-228.

23. Rodero L, Cuenca-Estrella M, Cordoba S, Cahn P, Davel G, Kaufman S, et al. Transient fungemia caused by an amphotericin B-resistant isolate of Candida haemulonii. J Clin Microbiol 2002; 40: 2266-2269.

24. Taverna CG, Cordoba S, Isla G, Fernandez N, Garcia S, Mazza $\mathrm{M}$, et al. First case report of bloodstream infection due to a Candida species closely related to the novel species Candida pseudonigosa. J Clin Microbiol 2012; 50: 2165-2169.

25. Li J, Xu YC, Bai FY. Candida pseudonugosa sp. nov., a nove yeast species from sputum. J Clin Microbiol 2006; 44: 4486-4490.

26. Quindós $\mathrm{G}$. Epidemiology of candidaemia and invasive candidiasis. A changing face. Rev lberoam Micol 2014; 31: 42-48.

27. Quindós G. Candidiasis y otras infecciones humanas asociadas a biopelículas microbianas. Rev Semáforo 2014; 57: 53-55.

28. Pappas PG, Kauffman CA, Andes D, Benjamin DK, Jr. Calandra TF, Edwards JE, Jr., et al. Clinical practice guidelines for the management of candidiasis: 2009 update by the Infectious Diseases Society of America. Clin Infect Dis 2009; 48: 503-535.

29. Cauda R. Candidaemia in patients with an inserted medical device. Drugs 2009; 69 Suppl I: 33-38.

30. Osmon DR, Berbari EF, Berendt AR, Lew D, Zimmerli W, Steckelberg JM, et al. Diagnosis and management of prosthetic joint infection: clinical practice guidelines by the Infectious Diseases Society of America. Clin Infect Dis 2013; 56: el-e25.

31. European Committee on Antimicrobial Susceptibility Testing. Antifungal Agents Breakpoint tables for interpretation of MICs. Basilea, Suiza. 20 I5. Disponible: http://munw. eucast.org/fileadmin/src/media/PDFs/EUCAST_files/AFST/ Clinical_breakpoints/Antifungal_breakpoints_v_8.0_No vember_2015.pdf. Consultado: jun 2015.

32. Cuenca-Estrella M, Gomez-Lopez A, Cuesta I, Zaragoza O, Mellado E, Rodriguez-Tudela JL Frequency of voriconazole resistance in vitro among Spanish clinical isolates of Candida spp. According to breakpoints established by the Antifungal Subcommittee of the European Committee on Antimicrobial Susceptibility Testing. Antimicrob Agents Chemother 20 I I; 55: I794-1797.

33. Pfaller MA, Andes D, Diekema DJ, Espinel-Ingroff A, Sheehan D. Wild-type MIC distributions, epidemiological cutoff values and species-specific clinical breakpoints for fluconazole and Candida: time for harmonization of CLSI and EUCAST broth microdilution methods. Drug Resist Updat 20 I0; 13: 180-195.

34. Zuluaga Rodríguez A, de Bedout Gómez C, Agudelo Restrepo CA, Hurtado Parra $\mathrm{H}$, Arango Arteaga M, Restrepo Moreno Á, et al. Sensibilidad a fluconazol y voriconazol de especies de Candida aisladas de pacientes provenientes de unidades de cuidados intensivos en Medellín, Colombia (20012007). Rev lberoam Micol 2010; 27: 125-129.

35. Cordoba S, Vivot W, Bosco-Borgeat ME, Taverna C, Szusz W, Murisengo O, et al. Species distribution and susceptibility profile of yeasts isolated from blood cultures: results of a multicenter active laboratory-based surveillance study in Argentina. Rev Argent Microbiol 201 I; 43: 176-185.

36. Rodero L, Córdoba S, Vivot W, Campo M, Corfield $\mathrm{P}$, Olguín $\mathrm{C}$, et al. Método de difusión con discos para la determinación de sensibilidad a fluconazol en aislamientos de Candida spp. Rev Argent Microbiol 2006; 38: I55- 163.

37. Alexander BD, Johnson MD, Pfeiffer CD, jimenez-Ortigosa C, Catania J, Booker R, et al. Increasing echinocandin resistance in Candida glabrata: clinical failure correlates with presence of FKS mutations and elevated minimum inhibitory concentrations. Clin Infect Dis 2013; 56: 1724-1732.

38. Pham CD, lqbal N, Bolden CB, Kuykendall RJ, Harrison LH, Farley MM, et al. Role of FKS Mutations in Candida glabrata: $\mathrm{MIC}$ values, echinocandin resistance, and multidrug resistance. Antimicrob Agents Chemother 20 14; 58: 4690-4696.

39. Pfaller MA, Messer SA, Diekema DJ, Jones RN, Castanheira M. Use of micafungin as a surrogate marker to predict susceptibility and resistance to caspofungin among 3,764 clinical isolates of Candida by use of CLSI methods and interpretive criteria. J Clin Microbiol 2014; 52: 108- I|4.

40. Ferrer R, Gómez de la Cámara A, Jarque I, Quindós G, Tejerina T. Estudio comparativo multidisciplinar de las equinocandinas, anidulafungina, caspofungina y micafungina. Rev Act Farmacol Terp 2013; I : 146-159

41. Arendrup MC, Garcia-Effron G, Lass-Florl C, Lopez AG, Rodriguez-Tudela JL, Cuenca-Estrella M, et al. Echinocandin susceptibility testing of Candida species: comparison of EUCAST EDef 7.I, CLSI M27-A3, Etest, disk diffusion, and agar dilution methods with RPMI and isosensitest media. Antimicrob Agents Chemother 20 10; 54: 426-439.

42. Gómez S, García SM, de Bedout C, García AM. Análisis de perfil proteico de aislamientos clínicos de Candida guilliermondil sensibles y resistentes al fluconazol. Infectio 20 I I; 15: 20-24.

43. Cuenca-Estrella M, Alastruey-Izquierdo A, Gómez-López A Monzón A. Estudios de sensibilidad en levaduras. Actualización y novedades. Enferm Infecc Microbiol Clin 2013; 31, Supplement I: 53-58. 